\title{
Enhancing Web-based Learning by Sharing Affective Experience
}

\author{
Michael J. Lyons, Daniel Kluender, and Nobuji Tetsutani \\ ATR Media Information Science Labs \\ ATR Intelligent Robotics and Communication Labs \\ 2-2-2 Hikaridai, Keihanna Science City, Kyoto 619-0288, Japan \\ mlyons@atr.jp
}

\begin{abstract}
We suggest that the real-time visual display of affective signals such as respiration, pulse, and skin conductivity can allow users of web-based tutoring systems insight into each other's felt bodily experience. This allows remotely interacting users to increase their experience of empathy, or shared feeling and has the potential to enhance telelearning. We describe an implementation of such a system and present the results of a preliminary experiment in the context of a web-based system for tutoring the writing of Chinese characters.
\end{abstract}

\section{Introduction}

Telecommunication necessarily involves a degree of disembodiment. Even with audio, video, text and graphic links that are now commonly available with chat programs we do not have the rich, high bandwidth multi-sensorial exchange experienced in face-to-face interaction. A key deficit in machinemediated remote interaction is the much decreased level of non-verbal communication. Non-verbal signals such as gestures, facial expressions, and other forms of body language play an important role in implicit and affective aspects of communication. Skill in understanding and participating in these modes of interaction forms a significant component of social expertise.

Interactions in the context of education depend not only on the explicit exchange of information but also rely on implicit, affective modes of communication. Indication of salience, emphasis, understanding or misunderstanding, interest, boredom, acceptance, questioning, difficulty, all rely at least partially on non-verbal communication. Telelearning will continue to suffer from limitations on non-verbal exchange for the immediately foreseeable future.
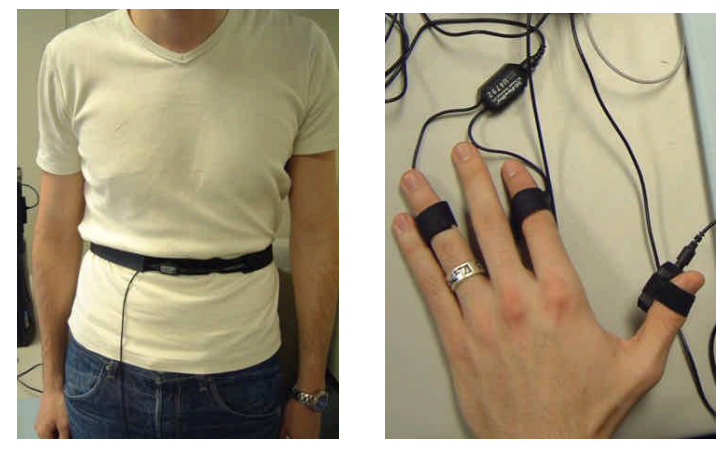

Figure 1 Sensors for respiration (abdomen), skin conductance (fingers), and blood volume pulse (thumb).

On the other hand, machine-mediation allows us to consider novel forms of non-verbal communication not previously experienced in natural face-to-face interactions. In this paper we propose the creation of artificial expressions of affective state and explore the consequences of sharing them in remote interaction.

Our hypothesis is that the increased embodiment of the interaction afforded by such artificial expressions can support the experience of empathy [8], or shared feeling, in remote interaction and that this can enhance educational interactions.

\section{System for Sharing Affective Experience}

Much of the prior work in affective computing has been aimed at human-computer interaction [6] and especially at machine recognition of affective state. In contrast, the present paper addresses computermediated human interaction. Rather than attempting to have machines classify emotional states we seek an effective way of gauging, representing, and communicating affective information in such a way as it may be easily interpreted by other humans. 


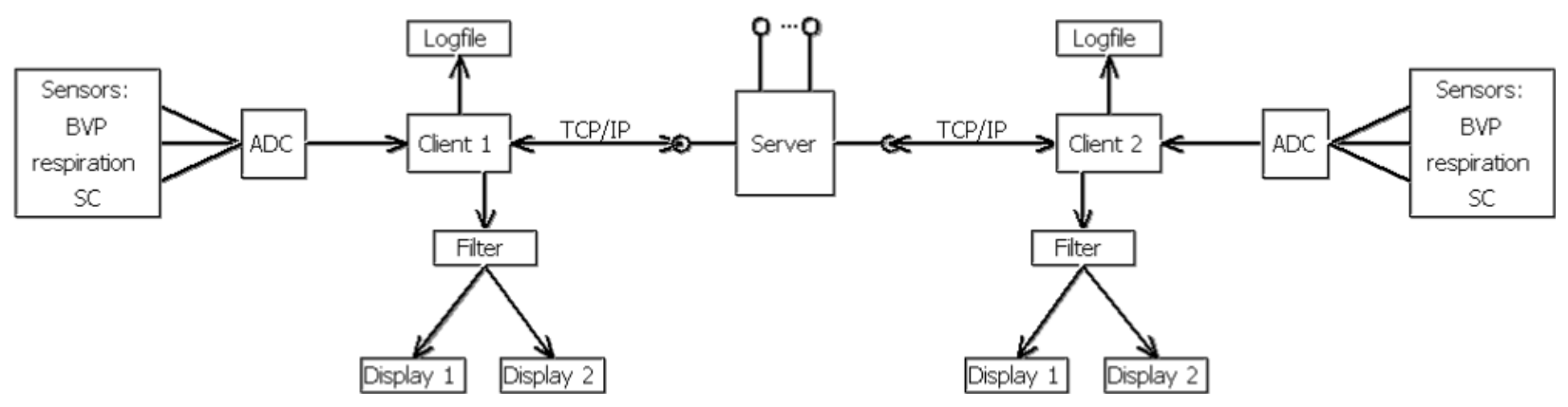

Figure 2. Schematic of the client server architecture used for the SAE system.

Our approach is motivated by a significant body of work on affect which suggests that high level appraisal of emotional episodes is highly dependent on context and past experience [7]. At a more basic level, consider a very widely studied indicator of emotional state, facial expression. Numerous studies of a constrained version of the problem of automatic facial expression recognition have shown some success [3, 5], however nearly all of these studies do not attempt to deal with the actual physical complexity of the problem (e.g. [4]) let alone even more difficult aspects such as context. Our more modest but practically realistic aim in this work is to provide channels to allow humans to gauge aspects of core affect [7] (for example, stress or arousal).

The design of the system involves several components. First, we use a set of non-intrusive wearable sensors which gauge the affective state of the wearer. Three robustly measurable variables, respiration, pulse, and skin conductance are used. We implement a client/server architecture for sharing this information over the internet in a transparent and user-friendly fashion. The signals are visualized intuitively in such a way that they can be understood without focal attention and with little learning. The shared affective experience (SAE) displays are intended to act as computer-mediated artificial expressions which give insight to the users felt affective experience. The meaning of these artificial expressions is intended to be learned constructively through social interaction with other users [10]. We study such interaction in the context of a specific learning task: a web-based tutoring environment for writing Chinese characters.

\section{Implementation}

\subsection{Sensors}

Respiration, blood volume pulse, and skin conductance were chosen because they can be measured robustly in a non-invasive fashion using readily available equipment. These signals are influenced by level of stress or arousal and are amongst the most widely used in "affective computing" [6].

The respiration $(\mathbf{R})$ sensor is worn around the chest or abdomen (Figure 1). It produces a voltage in response to expansions or contractions of the abdomen or chest in breathing. Breathing may be roughly characterized by rate (cycles per minute) and depth (or amplitude) as well as pauses in the motion when the breathing is irregular or stops. These variables are all related to affective state. For example, respiration rate increases and breathing becomes shallower with arousal, whereas with relaxation the breathing slows and deepens. Irregular breathing can result from tense states.

The blood volume pulse (BVP) sensor is worn on the tip of a finger or thumb (Figure 1). An infrared LED and light sensor are used to measure the reflectance near the surface of the skin. The amount of light reflected is a function of the volume of blood in the capillaries which varies with the pulse. The signal therefore depends on the pulse rate and blood pressure. A rise in the rate of the beating pulse and increase in amplitude correspond to arousal. 


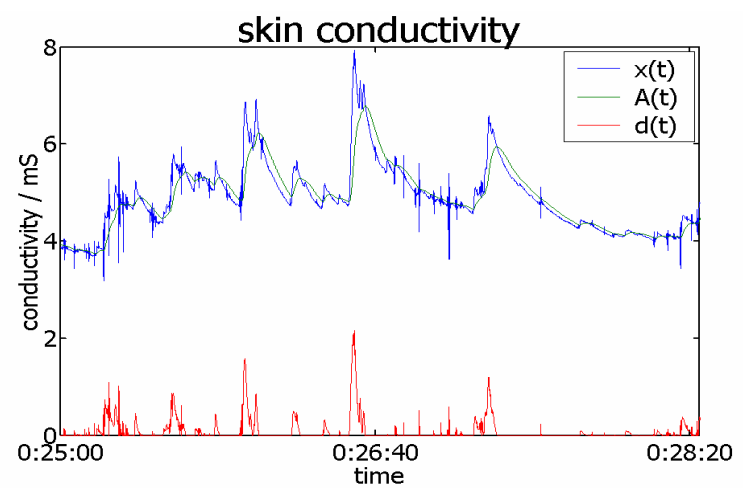

Figure 3. Time series of skin conductivity raw and high-pass filtered signals.

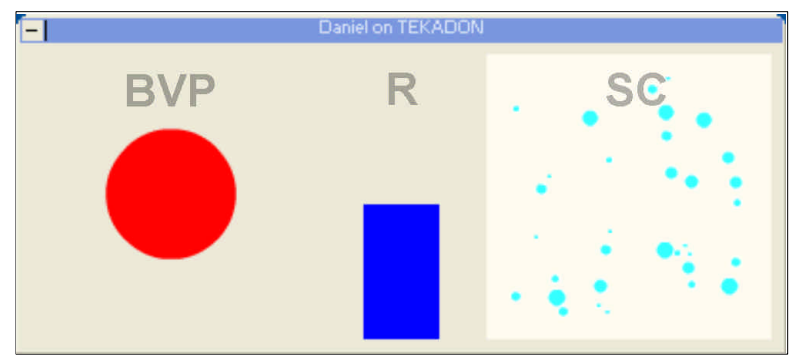

Figure 4 Shared Affective Experience (SAE) Displays

The skin conductance (SC) sensor is worn on two fingers of one hand, usually the same hand that the BVP sensor is worn on (Figure 1). This measures the electrical conductance of the skin. The SC signal measures the electrodermal response. This is readily demonstrated by the "startle response" - a small but definite jump in SC is seen in response to an unexpected noise or other stimulus. Changes in affect also result in a change in SC.

\section{2. $2 \mathrm{~A} / \mathrm{D}$ converter}

The Procomp + system (Thought Technologies Ltd.) is used to convert analogue voltages from the sensors to digital signals that are sent to the computer via the RS-232 serial port. This is a medical grade device that is intended for use in clinical work and in biofeedback training. It samples all three signals at a rate of $20 \mathrm{~Hz}$.

The system is connected to the serial port via a fiberoptic cable ensuring complete electrical isolation from the computer.

\section{3 Client/Server System}

A client process running on the local computer receives the samples from the three signals and sends all data to a broadcasting server using the TCP/IP protocol. A schematic of the client/server architecture of the system is shown in Figure 2. The server sends all received data to every client (hence the name broadcasting server) so each client can access all other clients sensor data. A single message containing a single sample from a sensor is 75 bytes in length (including some error protection overhead). With the sampling rate of $20 \mathrm{~Hz}$ this results in a total traffic rate of $36 \mathrm{kbits} / \mathrm{s}$ from each client. Our experiments took place on a 100 Mbit local area network. A clientto-client mean transmission time of $19.8 \mathrm{~ms}$ (standard deviation $12.8 \mathrm{~ms}$ ) was measured. This latency is not noticeable under the conditions of the experiment.

\section{4 Signal processing}

Little or no signal processing is applied to the data from the sensors. Simple linear processing is applied to the SC signal to remove the slowly decaying baseline of the electrodermal response (see the top line in Figure 3). We high-pass filter the SC signal with an IIR filter. Effectively, from each sample $x(i)$ the processed signal d(i) is calculating a weighted average of all previous samples:

$$
\begin{gathered}
d(i)=x(i)-A(i) \\
A(i)=A(i-1) * 0.995+x(i)+0.005
\end{gathered}
$$

The lower trace of Figure 3 shows the high-pass filtered SC signal. The peaks of this signal correspond to electrodermal events. A higher density of these events indicates a higher rate of sweating corresponding to greater arousal or level of stress.

\section{5 Display}

The three physiological signals are visualized by using a direct and intuitive display. Careful planning underlies the simple design of the SAE display shown in Figure 4. Two concepts guided our design. One is the idea of creating a visual metaphor. A metaphor is a means, in this case graphical, of expressing one kind of experience in terms of another [2]. The specific shapes, colours, and motions of the dynamic graphical displays were inspired by Arnheim's work on visual thinking [1] in that the dynamic features of the displays mirror the signals they represent. For example, as the chest or abdomen rises with an inbreath the blue column rises. This suggests 
identification of the internal experience of that physiological phenomenon with its concordant visual display. These considerations illustrate why we considerate it important to process the signals as little as possible: salient dynamic characteristics corresponding to felt bodily experiences are preserved.

A further reason for the visual simplicity and intuitiveness of the display is that it allows the information to be taken in at a quick glance, avoiding

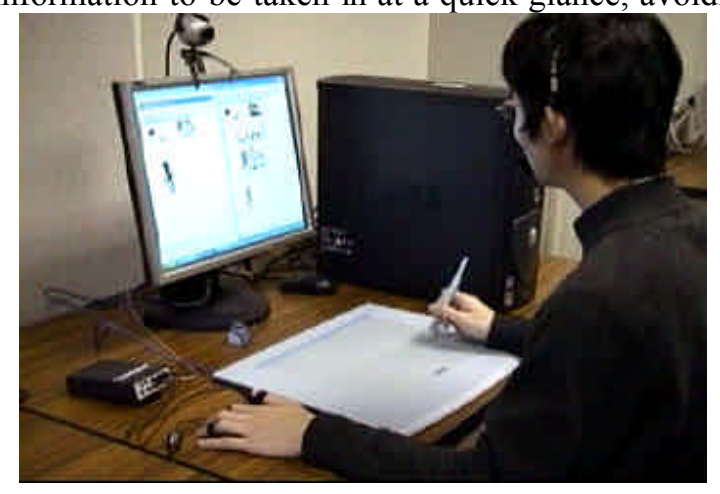

heavy cognitive demands for processing the artificial expressions. This is necessary if the display is to be used implicitly, in the periphery, as with the peripheral interfaces described by Weiser and Brown on in their work on calm technology [11].

Each graphic is motivated by the physiological function that it represents. The respiration signal is shown as a blue column, with increased height of the column corresponding to increased stretch of the $\mathbf{R}$

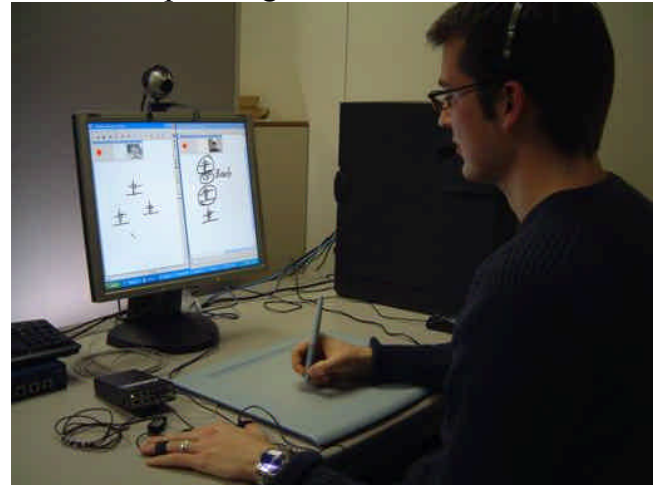

Figure 5. Web-based kanji tutoring platform.

sensor. The slowly rising and falling column is meant to suggest a lung inflating and deflating with the breathing cycle.

The value from the BVP sensor is displayed as the radius of a red circle. With the beating of the pulse the red circle expands and contracts like a beating heart.

The processed SC signal, d(i) in the equation above, is mapped to the probability of a blue circle suddenly expanding in size in a two dimensional display. This is suggestive of a patch of perspiring skin. At low values of SC the number of suddenly expanding patches is low and activity of the blue circles is sporadic. As SC increases the number increases and eventually the patch floods with blue as if the skin is saturated with sweat.

\section{Kanji Tutoring Environment}

Can the affective SAE displays we implemented have a beneficial effect on computer mediated tutoring? Do users learn to interpret the SAE displays as metaphors for felt experience of a remote tutor or student? We examined these questions empirically by conducting a preliminary study in the context of a web-based platform for remote kanji tutoring.

Kanji are the Chinese characters used in written Japanese. This specific learning task was chosen because, at any given time, there are several beginning learners of Japanese as a second language at our laboratory. For these learners, a lesson in writing kanji is an attractive prospect.

The kanji tutoring environment consists of an audio link and shared whiteboards (Figures 5 and 6). The student and teacher each have a Wacom tablet and stylus to enter handwritten kanji to the whiteboards. There is no noticeable latency in the audio and whiteboard links.

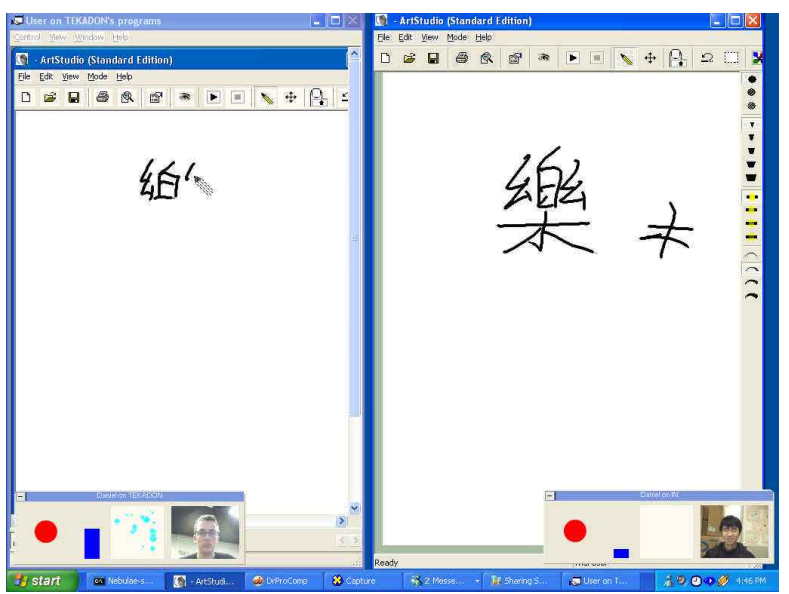

Figure 6. Shared whiteboards with SAE displays used in the experiment. 


\section{Preliminary Experiment}

The preliminary experiment took the form of a structured lesson. First, the basic strokes used in writing kanji were reviewed. Then the tutor introduced 5 kanji of an increasing level of difficulty. The lesson was followed by a short quiz. The entire lesson took between 30 minutes and 1 hour according to the individual pace of learning. Four unpaid, but highly motivated, volunteers took part in the experiment ( 3 students and 1 teacher). The primary aim of the experiment was to explore what meanings the visual metaphors we created could take on in a telelearning situation and whether users make use of the affective displays to gauge each others feelings.

We evaluated the interaction by interviewing the student and tutor separately after each session. They were asked whether they found the SAE displays

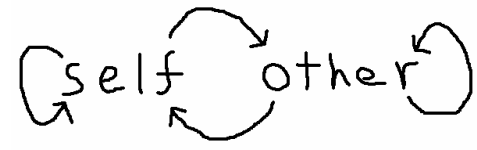

Figure 7. Self and Other

meaningful or useful for gauging their own emotional status of that of the other, during the task. Because both the task and the information displays were novel, the students generally stated that they were not yet able to make extensive use of the SAE displays during the lesson. However, one robust observation was that the skin conductance made the students more aware of their own emotional status.

The tutor is a member of our research group and so had greater familiarity with the interaction platform and physiological signal displays. The major observation of the tutor was that the skin conductance quickly became useful in pacing the lesson. By the tutor's account, excessive activity in the skin conductance was taken to imply that the level or speed of the lesson was too high for the student and needed to be relaxed.

\section{Conclusion}

Attending to felt bodily sensations can aid in recognizing and reducing stress. The hypothesis guiding the current study is that the SAE displays may increase empathy, the awareness both of our own and others' emotional states and that this could have numerous benefits for real-time web based tutoring, such as the kanji tutoring platform we studied.
An important design feature of the system we implemented is reciprocity: the affective displays are the same for tutor and student. This is a fundamental condition for the support of empathy or "shared feeling" [8]. One first identifies felt bodily experience with one affective display. This allows the user to infer the feelings of another by observation of the others affective display. Combined with contextual information, this can give users insight into how their actions influence another's felt experience and viceversa. This process, illustrated schematically in Figure 7, could, over the course of interaction, lead users to develop an intuitive sense of each others being.

The findings of our study in the context of a webbased kanji tutoring platform are consistent with our hypothesis that affective displays such as the ones we developed can engender the experience of empathy in online interaction.

\section{Acknowledgements}

We thank Gamhewage C. De Silva and Chi-Ho Chan for helpful interactions. This work was supported by the National Institute of Information and Communications Technology.

\section{References}

[1] Arnheim, R. Visual Thinking, University of California Press, Berkeley CA, 1969.

[2] Lakoff, G. and Johnson, M., Metaphors We Live By, University of Chicago Press, Chicago, 1980.

[3] Lyons, M.J., Budynek, J., and Akamatsu, S., Automatic Classification of Single Facial Images, IEEE PAMI 21(12): 1357-1362, 1999.

[4] Lyons, M.J., Campbell, R. et al., The Noh Mask Effect: Vertical Viewpoint Dependence of Facial Expression Perception, Proc. Roy. Soc. Lond. B 267: 2239-2245, 2000.

[5] Pantic, M. and Rothkrantz, L.J.M., Automatic Analysis of Facial Expressions: The State of the Art, IEEE PAMI 22: 1424-1445, 2000.

[6] Picard, R. W. Affective Computing, MIT Press, Cambridge MA, 2000.

[7] Russell, J. A., Core Affect and the Psychological Construction of Emotion, Psychological Review, 110(1): 145-172, 2003.

[8] Thompson, E.. "Empathy and Consciousness", Journal of Consciousness Studies 8, No. 5-7: 1-32, 2001. 
[9] Varela, F., E. Thompson, and E. Rosch, The Embodied Mind: Cognitive Science and Human Experience, MIT Press, Cambridge MA, 1991.

[10] Vygotsky, L.S. Mind in Society: The Development of Higher Psychological Processes. Harvard University Press, 1980.
[11] Weiser, M. and J. S. Brown, "The Coming Age of Calm

Technology",http://www.ubiq.com/hypertext/weiser/acmfutu re2endnote.htm, 1996. 